\title{
Habitats and trophic relationships of Chironomidae insect larvae from the Sepotuba River basin, Pantanal of Mato Grosso, Brazil
}

\author{
Butakka, CMM. ${ }^{*}$, Grzybkowska, M. ${ }^{B}$, Pinha, GD. ${ }^{C}$ and Takeda, AM. ${ }^{D}$ \\ ${ }^{a}$ Research Centre in Limnology, Biodiversity and Etnobiology of Pantanal, Av. Santos Dumont, s/n., \\ Cidade Universitária, Bloco II, CEP 78200-000, Cáceres, MT, Brazil \\ ${ }^{b}$ Department of Ecology and Vertebrate Zoology, University of Lodz, Banacha 12/16, 90-237 Lodz, Poland \\ 'Postgraduate Course in Continental Aquatic Environments, State University of Maringá - UEM, \\ Av. Colombo, 5790, Cep 87020-900, Maringá, PR, Brazil \\ ${ }^{\mathrm{d}}$ Nupelia, Department of Biology, National Council of Scientific and Technological Development, State University of \\ Maringá - UEM, Av. Colombo, 5790, Cep 87020-900, Maringá, PR, Brazil 304692/2002-6 \\ *e-mail: cristinabutakka@yahoo.com.br
}

Received December 12, 2012 - Accepted February 26, 2013 - Distributed May 31, 2014

(With 4 figures)

\begin{abstract}
Benthic habitats are linked by physical processes and are essential elements in assessing of the distribution dynamics of Chironomidae dipteran insects and their role in aquatic ecosystems. This work presents results of distribution patterns of chironomids larvae in 38 sites that are abundant in the study site, inhabiting the substrate of the main river channel, rapids, tributary brook, floodplain lakes and reservoir along the Sepotuba River from its mouth at the Paraguay River to the headwater region. A total of 1,247 larvae was registered. The most abundant taxa were Polypedilum (Tripodura) sp. (25.2\%), Cricotopus sp.3 (23.0\%) and Tanytarsus sp. (15.0\%). Fissimentum desiccatum were found only in the reservoir; Fissimentum sp.2 and Tanytarsus cf. T. obiriciae sp. 2 in floodplain lakes, and Goeldichironomus sp. in the main channel. The low diversity of the sites S06 and S35 is caused by the near-exclusive presence of the species Cricotopus sp.3, alone or together with one or another taxon (Tanytarsus sp., Djalmabatista sp.3). Collectors-filterers represent $16 \%$, collectors-gatherers $15 \%$, predators $11 \%$ and scrapers only $1 \%$. The predators dominated in the secondary channel $\left( \pm 88 \mathrm{ind} / \mathrm{m}^{2}\right)$, corresponding to $40 \%$ of the total of this group. Cryptochironomus sp.2 (34\%) and Ablasbemyia gr. annulata $(26 \%)$ were the most abundant among the predators. The differences along the river course are decisive for the formation of distinct or discontinuous communities and the limits become obvious though the interrelations between the populations in the community, as for instance, competition for food and habitats.
\end{abstract}

Keywords: Chironomidae, functional trophic groups, diversity, Pantanal of Mato Grosso, floodplains.

\section{Hábitats e relações tróficas de insetos Chironomidae da bacia do Rio Sepotuba, Pantanal de Mato Grosso, Brasil}

\begin{abstract}
Resumo
Hábitats bênticos estão ligados por processos físicos e são elementos essenciais na avaliação da dinâmica de distribuição de insetos dípteros Chironomidae e seu papel em ecossistemas aquáticos. Este trabalho apresenta os resultados dos padrões de distribuição de larvas de Chironomidae em 38 locais que são abundantes na área de estudo, habitando o substrato do canal principal do rio, cachoeiras, córregos tributários, lagoas de planície de inundação, e reservatórios ao longo do Rio Sepotuba de sua desembocadura ao Rio Paraguai e na região de cabeceira. Um total de 1,247 larvas foi registrada. O táxons mais abundantes foram Polypedilum (Tripodura) sp. (25.2\%), Cricotopus sp.3 (23.0\%) e Tanytarsus sp. (15.0\%). Fissimentum desiccatum foi encontrado somente no reservatório; Fissimentum sp.2 e Tanytarsus cf. T. obiriciae sp.2 nas lagoas de planície de inundação, e Goeldichironomus sp. no canal principal. A baixa diversidade dos locais S06 e S35 foi causada pela exclusiva presença da espécie Cricotopus sp.3, sozinha ou junta com um ou outro táxon (Tanytarsus sp., Djalmabatista sp.3). Coletores-filtradores representaram 16\%, coletores-catadores $15 \%$, predadores $11 \%$ e raspadores somente $1 \%$. Os predadores dominaram no canal secundário $\left( \pm 88 \mathrm{ind} / \mathrm{m}^{2}\right)$, correspondendo a $40 \%$ do total deste grupo. Cryptochironomus sp.2 (34\%) e Ablasbemyia gr. annulata (26\%) foram os mais abundantes entre os predadores. As diferenças ao longo do curso do rio foram decisivas para a formação de comunidades distintas e descontínuas e os limites tornam óbvios pertinentes às interrelações entre as populações na comunidade, como por exemplo, competição por alimento e hábitat.
\end{abstract}

Palavras-chave: Chironomidae, grupos funcionais tróficos, diversidade, Pantanal de Mato Grosso, planícies de inundação. 


\section{Introduction}

Continuous loss of habitat in recent decades has enhanced studies on the survey of species richness, in order to prioritise the conservation of areas with high diversity. A definition of biodiversity includes all terrestrial and freshwater organisms - including plants, animals, and microbes - at scales ranging from genetic diversity within populations, to species diversity, to community diversity across landscapes (Sala et al., 2000). In this context, invertebrates are recognised as important components of biodiversity (Kim, 1993; Kremen et al., 1993; Oliver and Beattie, 1996; Yen and Butcher, 1997), because they are important in all ecosystems in terms of species numbers and biomass.

The large extension of Brazilian territory causes that much of its diversity is still unknown. Thus, this work constitutes the first register of the Chironomidae community in the Sepotuba River, an important tributary of the Paraguay River north of the Pantanal. Its discharge contributes to the wave-like inundation patterns so famously transversing the Pantanal from north to south when the rainy season starts and which gives rise to the wetlands ecology.

In the Paraguay River and its tributaries, Chironomidae larvae are potentially important in the food chain (Wantzen and Junk, 2005) and abundant aquatic insects found in benthic communities (Stur et al., 2000, 2006; Aburaya and Callil, 2007). In this basin, research has been conducted to understand ecological aspects (Da Silva et al., 2001; Wantzen and Junk, 2005), aquatic invertebrates (De Drago et al., 2004; Marchese et al., 2005; Wantzen and Wagner, 2006), and biodiversity (Alho et al., 2003; Junk et al., 2006).

The role of food as a controlling factor of the population dynamics of Chironomidae larvae was recognised by Merrit and Wallace (1981), Pinder (1995) among others. In freshwaters of Brazil, the importance of aquatic invertebrates, especially Chironomidae, has often been demonstrated and some research has been done to understand their distribution in running water ecosystems (Trivinho-Strixino et al., 2000; Higuti and Takeda, 2002), ecological (Sanseverino and Nessimian, 2001; Henriques-Oliveira et al., 2003; Sanseverino and Nessimian, 2008) and taxonomic aspects (Trivinho-Strixino and Sanseverino, 2003; Roque and Trivinho-Strixino, 2003).

If the studies about species density and repartition of the diet between members of numerous Chironomidae larvae produce important information about distribution and their function (Trivinho-Strixino and Strixino, 1998; Nessimian et al., 1999), to study a new area will increase the knowledge about the distribution patterns of this group.

We studied the structure of the Chironomidae community in different habitats (main river channel, rapids, tributary brook, backwaters and reservoir) along the Sepotuba River and their trophic relationships, allowing us to detect the main factors that control the distribution patterns of this community.

\section{Study Area}

The catchment of the Sepotuba River is situated between $14^{\circ} 20^{\prime}$ to $16^{\circ} 00^{\prime} \mathrm{S}$ and $57^{\circ} 00^{\prime}$ to $58^{\circ} 40^{\prime} \mathrm{W}$, north of the city of Cáceres. It is part of the Upper Paraguay River basin, which is part of the transitional area between the realm of the Cerrado and of the Amazon forest (Ab'Saber, 1983). In the Pantanal floodplains there are many shallow floodplain lakes and lentic aquatic systems (Da Silva et al., 2001).

The Sepotuba River has as tributaries several blackwater streams, and floodplain-lakes with clear water, which support large quantities of aquatic macrophytes, including Eichhornia azurea, Pistia stratiotes, Salvinia spp. The hydrologic characteristics of the river are similar to those of the Paraguay River. Floods are caused by heavy rains in the upper parts of the basin, and propagate to the region of the Pantanal.

The study area comprises different aquatic systems, defined here as "main river channel", "rapids", "tributary brook", "floodplain lakes", and "reservoir" along the Sepotuba River from its mouth at the Paraguay River to the headwater region. 38 sampling sites were chosen along the river (Table 1; Figure 1).

\section{Material and Methods}

Collections were carried out with a Petersen grab $\left(0.0189 \mathrm{~m}^{2}\right)$ during February and March 2002 at 38 sampling sites, with the exception of sampling site S32, with three samples taken with a Surber sampler with an area of $0.09 \mathrm{~m}^{2}$. At each site four samples were taken, three for biological and one for sediment granulometry and organic matter content analyses. All material was fixed in the field in $70 \%$ alcohol.

Concurrently with the collections of biological material, water transparency, $\mathrm{pH}$, electric conductivity $(\mathrm{mS} / \mathrm{cm})$, dissolved oxygen $(\%)$ and water temperature $\left({ }^{\circ} \mathrm{C}\right)$ were measured. The granulometric texture was determined according to the Wentworth Scale (Wentworth, 1922). Organic matter content was determined from $10 \mathrm{~g}$ of sediment sample by incineration at $560^{\circ} \mathrm{C}$.

The samples for biological analysis were washed through a series of sieves with smallest mesh size $0.2 \mathrm{~mm}$. In the laboratory, samples were sorted under a stereoscopic microscope.

The Chironomidae larvae were fixed on slides with Euparal, separating the head capsule from the rest of the body. The taxa were identified using the keys of Epler (2001), Coffman and Ferrington Junior (2008), and Trivinho-Strixino (2011). The structure of the community was analysed based on the systematic composition, Shannon-Wiener diversity index (H') and Margalef's index of richness (Magurran, 2004).

The mounted slides were used to analyse the food items of the digestive tract, using the keys Bicudo and Bicudo (1970) and Parra and Bicudo (1995) and the larvae were fixed on slides with Euparal.

\subsection{Functional feeding groups}

Based on traditional functional feeding groups of Chironomidae taxa, we were able to indicate which of the taxa found are ordered (Table 2). For the characterisation 
Table 1. Study area with 38 sampling sites in different aquatic systems in the Sepotuba River basin.

\begin{tabular}{|c|c|c|}
\hline Site & Geographical coordinates & Environmental \\
\hline S01 & $15^{\circ} 59^{\prime} 59^{\prime \prime} \mathrm{S}-5^{\circ} 39^{\prime} 11^{\prime \prime} \mathrm{W}$ & $\begin{array}{l}\text { Floodplain lake - with riparian vegetation and aquatic macrophytes. } \\
\text { Connection of the Sepotuba and Paraguay River }\end{array}$ \\
\hline S02 & $15^{\circ} 59^{\prime} 23^{\prime \prime S}-57^{\circ} 39^{\prime} 35^{\prime \prime} \mathrm{W}$ & Mouth of the Sepotuba River - narrow channel \\
\hline $\mathrm{S} 03$ & $15^{\circ} 58^{\prime} 55^{\prime} \mathrm{S}-57^{\circ} 39^{\prime} 45^{\prime \prime} \mathrm{W}$ & $\begin{array}{l}\text { Main channel of the Sepotuba River - semi-lotic with many aquatic } \\
\text { macrophytes }\end{array}$ \\
\hline S04 & $15^{\circ} 57^{\prime} 48^{\prime \prime} \mathrm{S}-57^{\circ} 39^{\prime} 00^{\prime \prime} \mathrm{W}$ & $\begin{array}{l}\text { Floodplain lake on the left bank of the Sepotuba River - riparian } \\
\text { vegetation and macrophytes (Eichhornia azurea) }\end{array}$ \\
\hline $\mathrm{S} 05$ & $15^{\circ} 57^{\prime} 31^{\prime \prime S}-57^{\circ} 39 ’ 30^{\prime \prime W}$ & Main channel of the Sepotuba River \\
\hline S06 & $15^{\circ} 57^{\prime} 40^{\prime \prime S}-57^{\circ} 38^{\prime} 51^{\prime \prime} \mathrm{W}$ & Floodplain lake on the right bank of the Sepotuba River \\
\hline S07 & $15^{\circ} 50^{\prime} 25^{\prime \prime} \mathrm{S}-57^{\circ} 37^{\prime} 23^{\prime \prime} \mathrm{W}$ & Main channel of the Sepotuba River \\
\hline S08 & $15^{\circ} 45^{\prime} 38^{\prime \prime} \mathrm{S}-5^{\circ} 39^{\prime} 30^{\prime \prime} \mathrm{W}$ & Small secondary channel of the Sepotuba River on the right bank \\
\hline S09 & $15^{\circ} 45^{\prime} 55^{\prime \prime} \mathrm{S}-5^{\circ} 39^{\prime} 19^{\prime \prime} \mathrm{W}$ & $\begin{array}{l}\text { Floodplain lake with macrophytes on the left bank of the Sepotuba } \\
\text { River }\end{array}$ \\
\hline $\mathrm{S} 10$ & $15^{\circ} 44^{\prime} 12^{\prime} \mathrm{S}-57^{\circ} 42^{\prime} 15^{\prime \prime} \mathrm{W}$ & $\begin{array}{l}\text { Secondary channel of the Sepotuba River - meandering on the right } \\
\text { bank, with strong current. Many branches and logs in the water }\end{array}$ \\
\hline $\mathrm{S} 11-\mathrm{S} 17$ & $\begin{array}{l}15^{\circ} 43^{\prime} 51^{\prime \prime} \mathrm{S}-57^{\circ} 41^{\prime} 19^{\prime \prime} \mathrm{W} / \\
15^{\circ} 39^{\prime} 25^{\prime \prime} \mathrm{S}-57^{\circ} 40^{\prime} 55^{\prime \prime} \mathrm{W}\end{array}$ & Main channel of the Sepotuba River \\
\hline $\mathrm{S} 18$ & $15^{\circ} 28^{\prime} 48^{\prime \prime S}-57^{\circ} 41^{\prime} 03^{\prime \prime W}$ & Small darkwater stream on the right bank of the Sepotuba River \\
\hline $\mathrm{S} 19, \mathrm{~S} 20$ & $\begin{array}{l}15^{\circ} 37^{\prime} 12^{\prime \prime} \mathrm{S}-57^{\circ} 40^{\prime} 48^{\prime \prime} \mathrm{W} / \\
15^{\circ} 35^{\prime} 18^{\prime \prime} \mathrm{S}-57^{\circ} 41^{\prime} 12^{\prime \prime} \mathrm{W}\end{array}$ & Main channel of the Sepotuba River \\
\hline $\mathrm{S} 21$ & $15^{\circ} 29^{\prime} 45^{\prime \prime} \mathrm{S}-57^{\circ} 43^{\prime} 14^{\prime \prime} \mathrm{W}$ & Humid grassland (vereda) on the right bank of the Sepotuba River \\
\hline $\mathrm{S} 22$ & $15^{\circ} 30^{\prime} 51^{\prime} \mathrm{S}-57^{\circ} 42^{\prime} 55^{\prime \prime} \mathrm{W}$ & Small darkwater stream on the right bank of the Sepotuba River \\
\hline $\mathrm{S} 23, \mathrm{~S} 24$ & $\begin{array}{l}15^{\circ} 08^{\prime} 22^{\prime \prime} \mathrm{S}-57^{\circ} 41 ' 23 ” \mathrm{~W} / \\
15^{\circ} 09^{\prime} 50^{\prime \prime} \mathrm{S}-57^{\circ} 41 ' 20^{\prime \prime} \mathrm{W}\end{array}$ & Main channel of the Sepotuba River \\
\hline $\mathrm{S} 25$ & $15^{\circ} 10^{\prime} 15^{\prime \prime} \mathrm{S}-57^{\circ} 41^{\prime} 45^{\prime \prime} \mathrm{W}$ & $\begin{array}{l}\text { Small darkwater stream on the right bank of the Sepotuba River, with } \\
\text { logs in the water }\end{array}$ \\
\hline S26 & $15^{\circ} 12^{\prime} 30^{\prime \prime S}-57^{\circ} 40^{\prime} 55^{\prime \prime} \mathrm{W}$ & Small clearwater stream on the left bank of the Sepotuba River \\
\hline S27 & $15^{\circ} 16^{\prime} 19^{\prime \prime S}-57^{\circ} 42^{\prime} 36^{\prime \prime} \mathrm{W}$ & $\begin{array}{l}\text { Small darkwater stream (Riacho Doce) on the right bank of the } \\
\text { Sepotuba River }\end{array}$ \\
\hline $\mathrm{S} 28, \mathrm{~S} 29$ & $\begin{array}{l}15^{\circ} 18^{\prime} 14^{\prime \prime} \mathrm{S}-57^{\circ} 42^{\prime} 16^{\prime \prime} \mathrm{W} / \\
15^{\circ} 20^{\prime} 00^{\prime \prime} \mathrm{S}-57^{\circ} 433^{\prime} 39^{\prime \prime} \mathrm{W}\end{array}$ & Main channel of the Sepotuba River \\
\hline $\mathrm{S} 30$ & $14^{\circ} 47^{\prime} 17^{\prime} \mathrm{S}-58^{\circ} 04^{\prime} 31^{\prime \prime} \mathrm{W}$ & $\begin{array}{l}\text { Juba II reservoir - upriver and close to the reservoir, with slightly dark } \\
\text { water, and many standing logs (remnants of inundated vegetation) }\end{array}$ \\
\hline S31 & $14^{\circ} 47^{\prime} 53^{\prime \prime S}-58^{\circ} 03 ’ 41^{\prime \prime} \mathrm{W}$ & $\begin{array}{l}\text { Juba II reservoir - downriver of the reservoir, with slightly dark water, } \\
\text { and many standing logs }\end{array}$ \\
\hline $\mathrm{S} 32$ & $14^{\circ} 48^{\prime} 15^{\prime} \mathrm{S}-57^{\circ} 59^{\prime} 11^{\prime} \mathrm{W}$ & Juba 0 - rapids upriver of the reservoir \\
\hline $\mathrm{S} 33$ & $14^{\circ} 37^{\prime} 54^{\prime \prime S}-57^{\circ} 42^{\prime} 18^{\prime \prime} \mathrm{W}$ & Sapo River - below the rapids \\
\hline $\mathrm{S} 34$ & $14^{\circ} 36^{\prime} 10^{\prime \prime S}-57^{\circ} 45^{\prime} 16^{\prime \prime} \mathrm{W}$ & Sapo River - above the rapids \\
\hline $\mathrm{S} 35$ & $14^{\circ} 37^{\prime} 23^{\prime \prime S}-57^{\circ} 41^{\prime} 47^{\prime \prime} \mathrm{W}$ & $\begin{array}{l}\text { Waterfall "Salto das Nuvens" formed by the Sepotuba River, with a } \\
\text { sandbar below the waterfall }\end{array}$ \\
\hline S36 & $14^{\circ} 29^{\prime} 16^{\prime \prime S}-57^{\circ} 35^{\prime} 28^{\prime \prime} \mathrm{W}$ & $\begin{array}{l}\text { Main channel of the Sepotuba River - in the region of the rapids, } \\
\text { Solange road }\end{array}$ \\
\hline $\mathrm{S} 37$ & $14^{\circ} 28^{\prime} 10^{\prime \prime S}-57^{\circ} 27^{\prime} 15^{\prime \prime} \mathrm{W}$ & Main channel of the Sepotuba River - rapids, below the bridge \\
\hline $\mathrm{S} 38$ & $14^{\circ} 28^{\prime} 25^{\prime \prime} \mathrm{S}-57^{\circ} 31^{\prime} 39^{\prime \prime} \mathrm{W}$ & Stream "Riacho do Heraldo" \\
\hline
\end{tabular}

of the habitat preference of each taxon, the site with the greatest number of records of the taxa was used. After this, we analysed the gut content of the most abundant taxa (Table 3). Habitat types were differentiated according to the type of aquatic system (lotic - lentic) and the type of substrate.

The sampling units were surveyed for their spatial relationships. Using Spearman's correlation coefficient
( $r$ ) for the data of density and diversity, the associations between the species were inferred.

According to Baselga (2010), we calculated three measures of beta diversity, the Sorensen dissimilarity index $\left(\mathrm{b}_{S O R}\right)$, the Simpson dissimilarity index $\left(\beta_{S I M}\right)$ and the complementary of $b_{S I M}$ was called residuals, by a routine that we wrote in $\mathrm{R}$ ( $\mathrm{R}$ Foundation for Statistical Computing, 2011). The total beta diversity is represented by 


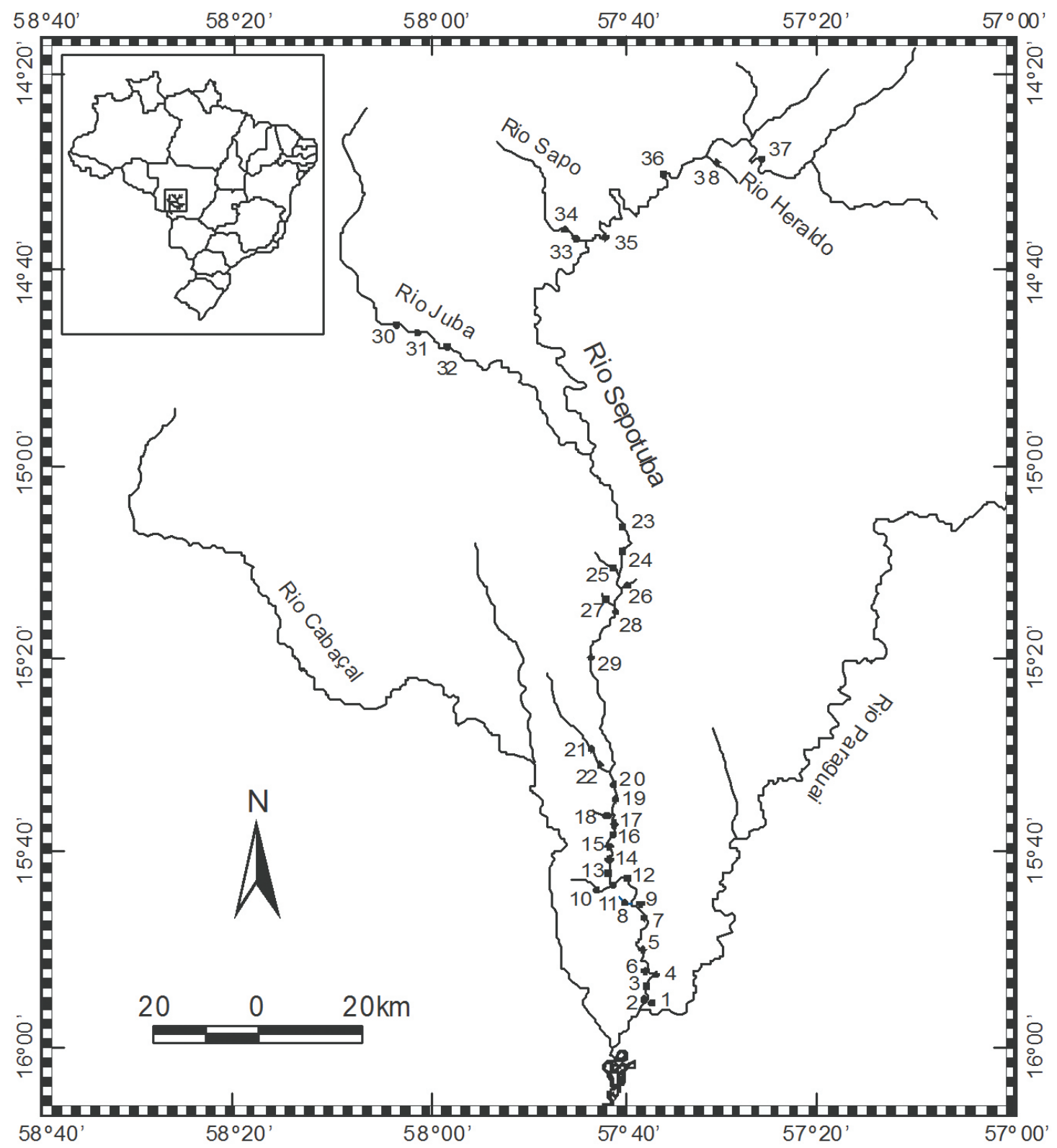

Figure 1. Map of the study area of the Sepotuba River with the sampling sites.

$\mathrm{b}_{S O R}$ and $\mathrm{b}_{S I M}$ is the species turnover part of beta diversity. The formulae that we used are as follows:

$\beta$ sor $=b+c /(2 a+b+c)$

\section{$\beta \operatorname{sim}=\min (b, c) /[a+\min (b, c)]$}

where $a$ is the number of species in both cells, $b$ is the number of species exclusive to the focal cell and $c$ is the number of species exclusive to the adjacent cell.

In order to evaluate the groups based on biological composition we calculated a cluster analysis by PC-Ord software (McCune \& Mefford, 1999).

\section{Results}

\subsection{Abiotic variables}

The water depth of the main channel varied from 0.2 $\mathrm{m}$ to $10.0 \mathrm{~m}$, while the dissolved oxygen varied between $19.5 \%$ and $115.2 \%$, and the $\mathrm{pH}$ between 5.00 and 7.47 (Table 4). In sampling sites with low water depth $(0.3 \mathrm{~m})$ the oxygen saturation reached its maximum. At the site with the greatest water depth $(10 \mathrm{~m})$, the saturation was $72.1 \%$ and the $\mathrm{pH}=6.06$.

Abiotic factors of 17 sampling sites in the main channel of the Sepotuba River showed some significant 
Table 2. Habitats and functional feeding groups of Chironomidae of the Sepotuba River Basin.

\begin{tabular}{|c|c|c|}
\hline Taxons & Habitat & $\begin{array}{c}\text { Functional } \\
\text { feeding group }\end{array}$ \\
\hline Chironomini Apedilum sp.1 & Stream - bottom sandy with gravel. & Collector-gatherer \\
\hline Beardius sp.3 & $\begin{array}{l}\text { Secondary channel and Stream - littoral and bottom sandy or } \\
\text { muddy; floodplain lake - bottom gravely-sandy }\end{array}$ & Collector-gatherer \\
\hline Chironomus $c f$. C. strenzkei & $\begin{array}{l}\text { Shallow floodplain lake - littoral with organic matter; } \\
\text { Channel, Stream and Reservoir - bottom rocky. }\end{array}$ & $\begin{array}{l}\text { Collector-gatherer; } \\
\text { shredder-herbivore }\end{array}$ \\
\hline Chironomus sp. & Channel - bottom sandy; floodplain lake -bottom with mud. & $\begin{array}{l}\text { Collector-gatherer; } \\
\text { shredder-herbivore }\end{array}$ \\
\hline Cryptochironomus sp. 2 & $\begin{array}{l}\text { Channel - central bottom rocky, littoral sandy; Stream - } \\
\text { bottom gravely-sandy }\end{array}$ & Predator \\
\hline Endotribelos sp.1 & $\begin{array}{l}\text { Channel - bottom sandy, littoral with gravel; Stream - bottom } \\
\text { gravely-sandy. }\end{array}$ & Collector-gatherer \\
\hline $\begin{array}{l}\text { Fissimentum } c f . \mathrm{F} \text {. } \\
\text { desiccatum }\end{array}$ & Reservoir - bottom with logs, littoral with macrophytes. & Collector-gatherer \\
\hline Fissimentum sp.2 & bottom with mud and much & Collector-gatherer \\
\hline Goeldichironomus sp. & Channel - bottom sandy. & Collector-gatherer \\
\hline Pelomus cf. P. psamophilus & $\begin{array}{l}\text { floodplain lake - bottom rocky or mu } \\
\text { matter; Channel - bottom rocky, litto }\end{array}$ & Collector-gatherer \\
\hline Lauterboniella $s p .1$ & Channel - bo & Collector-gatherer \\
\hline Phaenopsectra $s p .1$ & Channel - bot & Scraper \\
\hline Asheum sp.1 & $\begin{array}{l}\text { Channel - bottom rocky, littoral sandy; Lake (baía) - bottom } \\
\text { muddy. }\end{array}$ & $\begin{array}{l}\text { Shredder-herbivore, } \\
\text { Collector-gatherer }\end{array}$ \\
\hline Polypedilum (Tripodura) & $\begin{array}{l}\text { Channel and rapids -bottom rocky, littoral gravely-sandy; } \\
\text { Stream - bottom sandy; Reservoir -bottom with branches, } \\
\text { littoral with organic matter; Lake (baía) - bottom muddy. }\end{array}$ & $\begin{array}{l}\text { Shredder-herbivore, } \\
\text { Collector-gatherer }\end{array}$ \\
\hline Polypedilum $g r$ fallax & Secondary channel - bottom with mud. & $\begin{array}{l}\text { Shredder- } \\
\text { herbivore, } \\
\text { Collector-gatherer }\end{array}$ \\
\hline Polypedilum sp. & Main and secondary channel & $\begin{array}{l}\text { Shredder- } \\
\text { herbivore, } \\
\text { Collector-gatherer }\end{array}$ \\
\hline Endotribelos sp.2 & . & Collector-gatherer \\
\hline $\begin{array}{l}\text { Pseudochironomini } \\
\text { Aedokritus } s p .1\end{array}$ & Cha & Collector-gatherer \\
\hline Tanytarsini Caladomyia sp.1 & Cha & r-filterer \\
\hline Rheotanytarsus sp.1 & $\begin{array}{l}\text { floodplain lake - bottom muddy; Channel - bottom rocky, } \\
\text { littoral sandy. }\end{array}$ & Collector-filterer \\
\hline ina $s p .1$ & Cha & Collector-gatherer \\
\hline Tanytarsus cf. T. obiriciae & floodplain lake -bo & Collector-filterer \\
\hline Tanytarsus sp. & $\begin{array}{l}\text { el -bottom with mud; Channel - bottom } \\
\text { ttoral sandy. }\end{array}$ & Collector-filterer \\
\hline $\begin{array}{l}\text { Pentaneurini } \\
\text { Ablabesmyia grannulata }\end{array}$ & $\begin{array}{l}\text { Channel - bottom sandy with mud and leaves; Secondary } \\
\text { channel - bottom with mud. }\end{array}$ & $\begin{array}{l}\text { Predator, Collector- } \\
\text { gatherer }\end{array}$ \\
\hline Ablabesmyia (Karelia) & $\begin{array}{l}\text { Stream - bottom sandy with much organic material; Channel - } \\
\text { bottom sandy, littoral gravely-sandy. }\end{array}$ & $\begin{array}{l}\text { Predator, Collector- } \\
\text { gatherer }\end{array}$ \\
\hline Labrundinia sp.2 & hottom sandy with mud and leaves & Predator \\
\hline $\begin{array}{l}\text { Procladiini Djalmabatista } \\
\text { sp. } 3\end{array}$ & $\begin{array}{l}\text { floodplain lake - bottom muddy, littoral sandy; Channel - } \\
\text { bottom rocky. }\end{array}$ & Predator \\
\hline Procladius sp. & Stream - bottom rocky-sandy, littoral sandy. & Predator \\
\hline $\begin{array}{l}\text { Tanypodini Clinotanypus sp.1 } \\
\text { Othocladiinae }\end{array}$ & Channel - bottom rocky, littoral sandy. & Predator \\
\hline Corynoneura sp. & $\begin{array}{l}\text { floodplain lake - bottom muddy; Rapids -bottom rocky, littoral } \\
\text { sandy. }\end{array}$ & Collector-gatherer \\
\hline Thienemanniella sp. 3 & $\begin{array}{l}\text { Channel - bottom rocky, littoral gravely-sandy; Reservoir - } \\
\text { bottom rocky. }\end{array}$ & Collector-gatherer \\
\hline Cricotopus sp.3 & $\begin{array}{l}\text { Channel - bottom rocky-gravely, littoral sandy; Reservoir - } \\
\text { bottom rocky; Rapids - bottom gravely-sandy, littoral sandy. }\end{array}$ & $\begin{array}{l}\text { Shredder-herbivore, } \\
\text { Collector-gatherer }\end{array}$ \\
\hline
\end{tabular}


Table 3. Food items of the main species of Chironomidae larvae of the Sepotuba River basin. $(+++)$ abundant $(>100$ specimens), $(++)$ frequent (51-100 specimens), $(+)$ present (1-50 specimens).

\begin{tabular}{|c|c|}
\hline Systematic group & Food items \\
\hline Beardius cf. B. parcus & Bacillariophyceae $(+)$, detritus $(++)$ \\
\hline Chironomus cf. C. strenzkei & $\begin{array}{l}\text { Cymbella }(+++), \text { Gomphonema parvulum }(+), \text { G. gracilis }(+), \text { Pinnularia } \\
(+), \text { P. subcaptata }(+), \text { Encyonema }(+) \text { detritus }(+++)\end{array}$ \\
\hline Cryptochironomus sp.2 & $\begin{array}{l}\text { Anabaena }(+), \text { Aulacoseira granulata }(+) \text {, Aulacoseira sp. }(++), \text { Eunotia } \\
(+), \text { Eunotia cf. trigibba }(+), \text { Facus }(+) \text {, detritus }(+) .\end{array}$ \\
\hline Fissimentum $c f$. F. desiccatum & $\begin{array}{l}\text { Encyonema }(+) \text {, Eunotia }(+) \text {, E. sudetica }(+) \text {, Frustulia rhomboides }(+) \text {, } \\
\text { Navicula } s p .(+), \text { N. radiosa }(+) \text {, Synedra ulna }(+) \text {, detritus }(+++)\end{array}$ \\
\hline Goeldichironomus sp & Bacillariophyceae $(+)$, Cyanophyceae $(+)$, Detritus $(+)$. \\
\hline Pelomus cf. P. psamophilus & Frustulia rhomboides $(+)$ \\
\hline Polypedilum (Tripodura) & Bacillariophyceae $(++)$, Aulacoseira granulata $(+++)$ detritus $(+++)$ \\
\hline Phaenopsectra sp.1 & Diploneis (+), Nitzschia (+) \\
\hline Asheum sp.1 & Bacillariophyceae $(+)$, detritus $(+)$ \\
\hline Caladomyia sp.1 & $\begin{array}{l}\text { Bacillariophyceae }(+) \text {, Aulacoseira granulata }(+) \text {, Aulacoseira sp. }(+), \\
\text { detritus }(+)\end{array}$ \\
\hline Rheotanytarsus sp.1 & $\begin{array}{l}\text { Eunotia }(++), \text { Flagilaria }(+), \text { Frustulia }(+), \text { Gomphonema parvulum }(+) \text {, } \\
\text { Gomphonema sp. }(+), \text { Navicula }(+) \text {, Centralles }(+) .\end{array}$ \\
\hline Tanytarsus sp. & $\begin{array}{l}\text { Acnanthes minutissima }(+), \text { Acnanthes sp. }(+), \text { Aulacoseira }(+++), \\
\text { Cymbella }(+++) \text {, Eunotia }(++) \text {, Encyonema }(+) \text {, Cyanophyceae }(+) \text {, detritus } \\
(+) \text {, fungi spores. }\end{array}$ \\
\hline Ablabesmyia gr.annulata & $\begin{array}{l}\text { Achnantes }(+) \text {, Aulacoseira }(+) \text {, Eunotia }(+) \text {, E. } c f . \text { trigibba }(+) \text {, Frustulia } \\
(+) \text {, Gomphonema parvulum }(++) \text {, Navicula } s p .(+) \text {, Cyanophyceae }(+), \\
\text { exuves of chironomids }(+) \text {. }\end{array}$ \\
\hline Djalmabatista sp.3 & Bacillariophyceae $(+)$ \\
\hline Cricotopus sp. 3 & $\begin{array}{l}\text { Aulacoseira grannulata }(+++) \text {, Cymbella }(+++) \text {, Navicula sp. }(+), \\
\text { Centralles }(2) \text {, Encyonema }(+), \text { Encyonema minutum }(+) \text {, Encyonema } \\
\text { mesianum }(+), \text { Eunotia }(+++) \text {, Gomphonema parvalum }(++), \\
\text { Cyanophyceae }(++) \text {, detritus }(++) \text {, fungi spores. }\end{array}$ \\
\hline
\end{tabular}

correlations among each other (correlation coefficient of Spearman). There was a negative correlation between dissolved oxygen and water depth $(\mathrm{r}=-0.506, \mathrm{p}=0.038)$ and between dissolved oxygen and $\mathrm{pH}(\mathrm{r}=0.619, \mathrm{p}=0.008)$.

Another correlation in the main channel was recorded between the transparency and conductivity of the water $(\mathrm{r}=-0.573, \mathrm{p}=0.016)$. However, at a few sites of this habitat the lowest values of transparency ( 0.55 to 1.60 $\mathrm{m})$ coincided with greater values of the conductivity (45.3 to $25.5 \mathrm{mS} . \mathrm{cm}^{-1}$ ). Not surprisingly, in the substrate higher organic matter content was significantly correlated with higher amount of mud $(\mathrm{r}=0.686, \mathrm{p}=0.002)$.

At six sampling sites of the stream, the depth was positively correlated with the water transparency ( $\mathrm{r}$ $=0.882, \mathrm{p}=0.019$ ), and negatively with the presence of pebbles $(r=-0.882, p=0.019)$ and of very coarse sand $(r=$ $-0.819, p=0.020$ ). A greater number of correlations also appeared between the sediment types, as gravel and very fine sand $(\mathrm{r}=-0.941, \mathrm{p}=0.005)$, gravel and medium sand $(\mathrm{r}=0.880, \mathrm{p}=0.020)$, and medium sand and very fine sand $(\mathrm{r}=0.885, \mathrm{p}=0.018)$.

\subsection{Biotic variables: density and diversity}

Throughout Sepotuba River 1247 Chironomidae larvae were registered (Table 2). The most abundant taxa were
Polypedilum (Tripodura), Cricotopus sp.3, Tanytarsus sp. and Caladomyia sp. (Figure 2). Some taxa were found in only one type of habitat, e.g. Fissimentum cf. F. desiccatum in the reservoir, Fissimentum sp.2 and Tanytarsus cf. T. obiriciae in floodplain lakes, and Goeldichironomus $\mathrm{sp}$. in the main channel. Other taxa were encountered in several lotic habitats, e.g. Apedilum sp.1, Cryptochironomus cf. C. reshchikov, Endotribelos sp.1, Lauterborniella sp.1, Stempellina sp.1, Tanytarsus sp., Ablabesmyia gr. annulata, Clinotanypus sp.1. In the rapids, commonly encountered species were Cricotopus sp.3 at sites with bottom of pebbles and sand, and Corynoneura sp. on rocks and sandy littorals.

The sites with greatest density (Figure 3) were S36 (2249 ind $\left.\mathrm{m}^{-2} \pm 260.30\right)$ and $\mathrm{S} 38$ (1481 ind. $\left.\mathrm{m}^{-2} \pm 194.40\right)$. $P$. (Tripodura) was the morphotype with the greatest density on the 38 sites ( 80 ind. $\mathrm{m}^{-2} \pm 193$ ), followed by Cricotopus sp.3 (67 ind. $\mathrm{m}^{-2} \pm 240$ ) and Tanytarsus sp. (37 ind. $\mathrm{m}^{-2}$ \pm 146 ). Lowest densities were recorded at the sites S01 (12 ind. $\mathrm{m}^{-2} \pm 1.40$ ) and $\mathrm{S} 35$ (9 ind. $\mathrm{m}^{-2} \pm 1.51$ ), (Figure $3 \mathrm{~A}$ ). The taxa with the lowest density were Clinotanypus sp. (0.23 ind. $\left.\mathrm{m}^{-2} \pm 1.43\right)$, Fissimentum sp. $2\left(0.15\right.$ ind. $\left.\mathrm{m}^{-2} \pm 0.95\right)$ and Tanytarsus cf. T. obiriciae $\left(0.15\right.$ ind $\left.\mathrm{m}^{-2} \pm 0.95\right)$. At sampling sites S07 and S10 no larvae of Chironomidae were recorded. 
Table 4. Limnological characteristics of the sampled sites. D - Depth; T - temperature; DO - dissolved oxygen; Cond conductivity (mS/cm); P - pebbles; G - gravel; VCS - very coarse sand; CS - coarse sand; MS - medium sand; SF - fine sand; VFS - very fine sand; M - mud; OM - organic matter.

\begin{tabular}{|c|c|c|c|c|c|c|c|c|c|c|c|c|c|c|c|c|}
\hline Sites & D (m) & Secchi & $\mathbf{T}$ & DO & OD & Cond & pH & $\mathbf{P}$ & G & VCS & CS & MS & FS & VFS & M & OM \\
\hline S01 & 4,8 & 1.5 & 27.7 & 2.45 & 38.6 & 34.0 & 30 & 0.00 & 0.00 & 0.00 & 0.00 & 0.00 & 7.35 & 59.50 & 33.1 & 1.70 \\
\hline S02 & 5.0 & 1.2 & 26.9 & 3.48 & 43.9 & 25.8 & 11 & .00 & 0.00 & 0.00 & 0.70 & 16.97 & 33.35 & 39.51 & .46 & 4.17 \\
\hline S03 & 2.7 & 1.0 & 26.8 & 3.64 & 45.5 & 26.2 & 6.06 & 0.00 & 0.02 & 3.08 & 18.2 & 23.78 & 39.34 & 11.06 & 4.46 & 1.99 \\
\hline S04 & 4.5 & 1.5 & 27.3 & 3.39 & 42.7 & 25.9 & 6.03 & 0.00 & 0.00 & 0.00 & 0.72 & 14.07 & 38.96 & 26.94 & 19.3 & 10.9 \\
\hline S05 & 8.0 & 0.9 & 27.1 & 4.49 & 56.8 & 26.1 & 6.20 & 3.62 & 3.30 & 2.26 & 20.4 & 40.81 & 25.99 & 2.53 & 1.09 & 0.55 \\
\hline S06 & 7.0 & 0.9 & 27.4 & 4.64 & 58.4 & 26.4 & 6.08 & 0.00 & 0.00 & 0.00 & 0.10 & 0.16 & 77.44 & 14.76 & 7.54 & 4.29 \\
\hline S07 & - & 0.65 & 27.8 & 4.37 & 54.4 & 27.9 & 6.03 & - & - & - & - & - & - & - & - & - \\
\hline S08 & 2.7 & 1.6 & 28.2 & 2.80 & 35.9 & 25.5 & 6.03 & 21.8 & 25.1 & 9.16 & 6.71 & 11 & 19 & 1.49 & 4.57 & 15.4 \\
\hline S09 & 3.6 & 0.7 & 27.3 & 4.25 & 53.5 & 30.1 & 6.31 & 0.00 & 0.00 & 0.00 & 0.00 & 0.00 & 26.74 & 60.92 & 12.3 & 2.36 \\
\hline S10 & - & - & - & - & - & - & - & - & - & - & - & - & - & - & - & - \\
\hline S11 & 5.0 & 0.6 & 27.1 & 5.94 & 74.7 & 32.3 & 6.48 & 0.00 & 0.00 & 0.00 & 0.18 & 31.85 & 53.27 & 12.01 & 2.69 & 0.66 \\
\hline S12 & 4.5 & 0.5 & 27 & 5.89 & 74.1 & 31.9 & 6.41 & 0.00 & 0.00 & 0.06 & 1.92 & 35.80 & 51.49 & 8.70 & 2.02 & 1.29 \\
\hline S13 & 10 & 0.5 & 26.9 & 5.74 & 72.1 & 31.9 & 6.59 & 0.06 & 0.00 & 0.35 & 11.6 & 44.85 & 11.40 & 23.66 & 8.00 & 2.04 \\
\hline S14 & 10 & 0.5 & 27.1 & 6.20 & 77.7 & 31.8 & 6.60 & 0.00 & 0.00 & 0.00 & 0.00 & 9.37 & 30.89 & 52.47 & 7.27 & 2.68 \\
\hline S15 & 10 & 0.5 & 27.2 & 6.11 & 76.5 & 32.1 & 6.56 & 0.00 & 0.00 & 0.00 & 0.00 & 4.33 & 74.47 & 19.96 & 1.25 & 0.21 \\
\hline S16 & 5.5 & 0.7 & 28.0 & 6.64 & 84.5 & 30.2 & 6.97 & 0.00 & 0.00 & 0.00 & 0.18 & 0.56 & 35.06 & 61.10 & 3.11 & 0.62 \\
\hline S17 & 5.0 & 0.6 & 28.2 & 8.86 & 88.5 & 30.2 & 6.86 & 0.00 & 0.00 & 0.00 & 0.00 & 0.00 & 47.85 & 43.64 & 8.51 & 0.72 \\
\hline S18 & 1.5 & 0.7 & 27.3 & 1.57 & 19.5 & 30.7 & 5.85 & 0.00 & 0.00 & 0.00 & 0.14 & 0.47 & 52.78 & 41.89 & 4.72 & 1.16 \\
\hline S19 & 3.7 & 0.7 & 27.5 & 6.99 & 88.6 & 28.5 & 7.17 & 0.00 & 0.00 & 0.00 & 0.00 & 0.47 & 55.42 & 41.06 & 3.04 & 0.71 \\
\hline $\mathrm{S} 20$ & 3.5 & 0.6 & 27.7 & 7.09 & 89.7 & 29.7 & 7.03 & 0.40 & 0.24 & 0.50 & 2.50 & 6.52 & 40.88 & 45.17 & 3.80 & 3.14 \\
\hline $\mathrm{S} 21$ & 0.3 & 0.3 & 7 & 8 & 115.2 & 1 & 2 & & 0 & 0.00 & 0.34 & 3. & 31.43 & 45.29 & 19.5 & 3.21 \\
\hline $\mathrm{S} 22$ & 0.6 & 0.6 & & 2.95 & 37.7 & & & & 0 & 0.00 & 0.00 & & 37.25 & 45.30 & 13.2 & 0.96 \\
\hline $\mathrm{S} 23$ & 3.2 & 0.7 & 4 & 7.35 & 92.5 & 30.0 & 2 & 0 & 61 & 10.5 & 17.1 & 62 & 40.85 & 5.27 & 7.88 & 0.74 \\
\hline S24 & 3.7 & 0.6 & 27.6 & 7.36 & 93.3 & 30.8 & 7.01 & 0.08 & 0.00 & 0.00 & 0.00 & 1.56 & 55.91 & 38.09 & 4.35 & 0.43 \\
\hline $\mathrm{S} 25$ & 1.0 & 0.5 & 29.1 & 2.06 & 26.9 & 45.3 & 5.86 & 0.00 & 0.00 & 0.04 & 0.06 & 0.06 & 25.81 & 65.73 & 8.29 & 2.49 \\
\hline S26 & 1.2 & 0.7 & 26.2 & 5.44 & 67.4 & 24.5 & 6.68 & 0.00 & 0.04 & 0.00 & 0.06 & 1.22 & 53.89 & 36.08 & 8.70 & 2.38 \\
\hline $\mathrm{S} 27$ & 0.5 & 0.5 & 28.4 & 3.92 & 50.4 & 36.4 & 6.09 & 0.19 & 0.52 & 1.28 & 2.92 & 15.30 & 49.17 & 21.28 & 9.34 & 8.81 \\
\hline S28 & 3.0 & 0.5 & 28.4 & 6.88 & 88.6 & 30.8 & 6.98 & 3.00 & 0.00 & 0.00 & 0.00 & 0.24 & 66.89 & 27.71 & 2.16 & 0.48 \\
\hline S29 & 2.5 & 0.6 & 28.3 & 6.87 & 87.3 & 30.8 & 6.89 & 1.42 & 1.36 & 0.25 & 3.82 & 12.58 & 59.65 & 14.56 & 6.35 & 1.10 \\
\hline $\mathrm{S} 30$ & 3.0 & 3.0 & 25.4 & 7.76 & 94.6 & 32.0 & 5.60 & 0.00 & 0.57 & 0.30 & 3.03 & 4.25 & 69.67 & 8.86 & 13.3 & 8.83 \\
\hline S31 & 4.3 & 2.7 & 26.3 & 7.71 & 95.5 & 3.1 & 5.23 & 20.2 & 18.7 & 1.82 & 2.50 & 3.71 & 32.57 & 5.93 & 14.5 & 5.78 \\
\hline S32 & 0.3 & 0.3 & 26.2 & 7.68 & 95.4 & 5.6 & 5.00 & 0.00 & 0.08 & 0.00 & 0.00 & 0.00 & 69.06 & 27.98 & 2.89 & 0.48 \\
\hline S33 & 0.5 & 0.5 & 26.4 & 7.77 & 96.8 & 15.5 & 7.14 & 9.33 & 0.83 & 0.60 & 18.5 & 27.42 & 35.43 & 6.66 & 1.20 & 0.59 \\
\hline S34 & 0.4 & 0.4 & 26.2 & 7.29 & 89.8 & 14.8 & 6.98 & 56.1 & 13.4 & 2.38 & 1.77 & 3.07 & 13.95 & 5.19 & 4.01 & 12.2 \\
\hline S35 & 1.2 & 1.2 & 26.4 & 8.75 & 109.1 & 30.6 & 7.42 & 0.00 & 0.00 & 0.00 & 0.00 & 2.56 & 96.65 & 0.69 & 0.10 & 0.13 \\
\hline S36 & 2.8 & 1.2 & 26.9 & 4.98 & 62.4 & 34.8 & 6.45 & 0.00 & 0.00 & 0.00 & 0.12 & 1.37 & 67.93 & 28.75 & 1.83 & 0.26 \\
\hline S37 & 2.5 & 1.2 & 28.0 & 7.50 & 95.8 & 45.5 & 7.47 & - & - & - & - & - & - & - & - & - \\
\hline S38 & 0.2 & 0.2 & 28.2 & 3.55 & 45.5 & 15.4 & 6.10 & 32.9 & 7.99 & 3.47 & 6.90 & 14.34 & 18.97 & 11.48 & 3.90 & 2.15 \\
\hline
\end{tabular}

\subsection{Diversity index and species richness}

Among the sites the diversity had a characteristic variation (Figure 3B), with the highest diversity at the following sites: main channel S05 (0.86), floodplain lake S09 (0.85), streams S22 (0.83) and S26 (0.80). P. (Tripodura), Cryptochironomus sp.2, Tanytarsus sp., and $A$. gr. Annulata were recorded in sites with higher diversity. Sites with zero diversity were S06 and S35, where only the species Djalmabatista sp.3 and Cricotopus sp.3, were recorded. The indices of diversity showed major values for sites in the main channel (0.98 and 0.71), and floodplain lakes (0.89 and 0.74) (Table 5). However, the mean values of Margalef's richness index and the taxa richness were higher in the lotic systems (main channel and streams).

\subsection{Food items, density, diversity and correlations of functional feeding groups}

A great part of the larvae food was constituted by algae, including in the guts content of predators, like $A$. gr. annulata and Djalmabatista sp.3. The algae present in the guts content belong in their great majority to the class Bacillariophyceae and few to the class Cyanophyceae. 


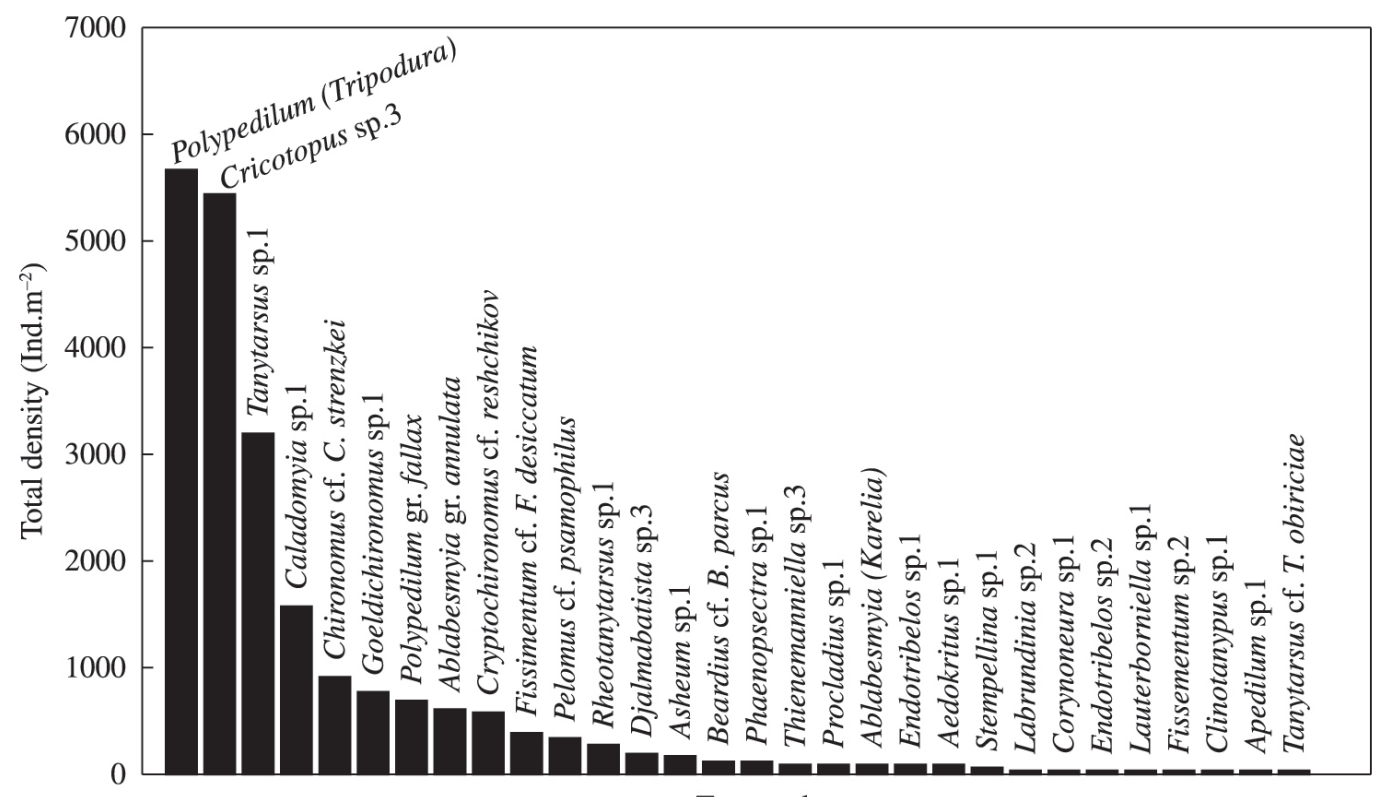

Taxa rank

Figure 2. Rank Abundance of Chironomidae morphotypes by Dominance curve.
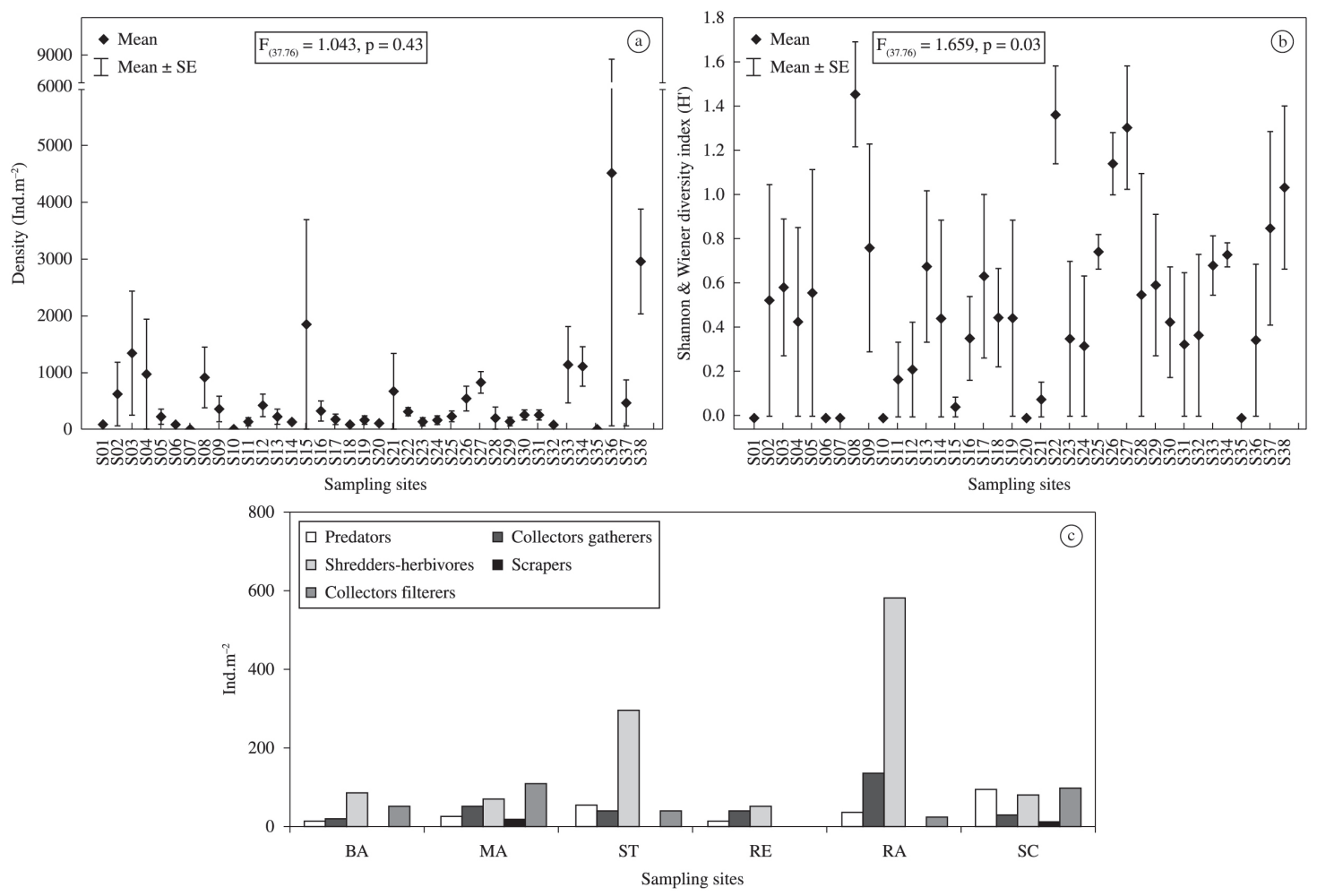

Figure 3. a- Mean density and standard error of Chironomidae larvae at 38 sampling sites in the Sepotuba River basin. b- Shannon-Wiener index of diversity (H') of Chironomidae larvae at the sampling sites of the Sepotuba River. c - Density according to functional feeding groups in different habitats of the Sepotuba River. BA=Floodplain lake, MA= Main channel, $\mathrm{ST}=$ Stream, $\mathrm{RE}=$ Reservoir, $\mathrm{RA}=$ Rapids, $\mathrm{SC}=$ Secondary channel. 
Table 5. Mean values of the Shannon-Wiener diversity index for functional feeding groups and Evenness, Margalef's richness index, and Taxa richness in the different habitats in different habitats. $\mathrm{BA}=$ Floodplain lake, MA= Main channel, $\mathrm{ST}=$ Stream, $\mathrm{RE}=$ Reservoir, $\mathrm{RA}=$ Rapids, $\mathrm{SC}=$ Secondary channel.

\begin{tabular}{|c|c|c|c|c|c|c|}
\hline & BA & MA & ST & RE & $\mathbf{R A}$ & SC \\
\hline \multicolumn{7}{|l|}{ Functional feeding groups } \\
\hline Predators & 0.12 & 0.64 & 0.46 & 0 & 0.39 & 0.22 \\
\hline Collectors-gatherers & 0.58 & 0.63 & 0.83 & 0.21 & 0.22 & 0.36 \\
\hline Shredders-herbivores & 0.54 & 0.47 & 0.27 & 0.42 & 0.26 & 0.23 \\
\hline Scrapers & 0 & 0 & 0 & 0 & 0 & 0 \\
\hline Collectors-filterers & 0.19 & 0.16 & 0.21 & 0 & 0.29 & 0 \\
\hline \multicolumn{7}{|l|}{ Taxa } \\
\hline Shannon-Wiener diversity & 0.89 & 0.98 & 0.74 & 0.67 & 0.53 & 0.78 \\
\hline Evenness & 0.74 & 0.71 & 0.57 & 0.79 & 0.47 & 0.78 \\
\hline Richness (Margalef) & 7.24 & 9.36 & 7.25 & 3.06 & 2.09 & 3.63 \\
\hline Taxa richness & 16 & 24 & 20 & 7 & 14 & 10 \\
\hline
\end{tabular}

The mean density values of functional feeding groups are shown in Figure 3C. Shredders-herbivores predominate in the major part of the systems, reaching $57 \%$ of the total. Collectors-filterers represent $16 \%$, collectors-gatherers $15 \%$, predators $11 \%$ and scrapers only $1 \%$. In the streams, the population density of shredders-herbivores reached 294 ind. $\mathrm{m}^{-2}( \pm 450.68)$; in the reservoir reached 51 ind. $\mathrm{m}^{-2}$ $( \pm 42.93)$ and, in the rapids 580 ind. $\mathrm{m}^{-2}( \pm 642.10)$, where $72 \%$ of the larvae belonged to Cricotopus sp.3.

Collectors-filterers represented $35 \%$ in the main channel (109 ind. $\left.\mathrm{m}^{-2}\right)$. Larvae of Tanytarsus sp. $\left(93\right.$ ind. $\left.\mathrm{m}^{-2}\right)$ made up the major portion among this group (40\%). Collectorsgatherers represented $45 \%\left(139\right.$ ind. $\left.\mathrm{m}^{-2} \pm 237.24\right)$ at the sites of the rapids and reached a population density of 49 ind. $\mathrm{m}^{-2}$ $( \pm 110.43)$ in the main channel. The predators dominated in the secondary channel $\left( \pm 88\right.$ ind. $\left.\mathrm{m}^{-2}\right)$, corresponding to $40 \%$ of the total of this group. Cryptochironomus sp. 2 (34\%) and $A$. gr. annulata (26\%) were the most abundant among the predators. Scrapers (Phaenopsectra) occurred only in the sampling site S20 in the main channel.

Comparisons of the structure of communities of Chironomidae larvae (density and composition) and abiotic factors in the main channel revealed few significant correlations, mainly with the type of sediment. The density of the species Caladomyia sp. showed a negative correlation with water temperature $(r=-0.562, \mathrm{p}=0.018)$ and a positive one with the coarseness of the sediment in the main channel, with the closest correlation with the sediment fraction "coarse sand" $(r=0.643, \mathrm{p}=0.005)$, followed by "very coarse sand" $(r=0.605, \mathrm{p}=0.010)$ and "medium sand" $(r=0.583, \mathrm{p}=0.014)$, while the correlation with "fine sand" was negative $(r=-0.512, \mathrm{p}=0.035)$. Another negative correlation to sediment parameters was found for Tanytarsus $\mathrm{sp}$. and muddy sediment $(\mathrm{r}=-0.671, \mathrm{p}=0.003)$.

Among the functional groups, the diversity of the collectors-gatherers was highest in the habitats "stream" $(0.83)$ and "main channel" (0.63) (see Table 3$)$. Characteristic taxon in this habitats were mainly Goeldichironomus sp., Pelomus cf. P. psamophilus and Caladomyia sp. The greatest diversity of predators was found in the main channel (0.64), where A. gr. annulata, Cryptochironomus cf. C. reshchikov, Djalmabatista sp. 3 and Labrundinia sp. 2 were the characteristic taxon for this habitat.

Collectors-filterers had low diversity, but were present in most of the habitats, e.g. Tanytarsus sp. and Rheotanytarsus sp.1, and sporadically Tanytarsus cf. T. obiriciae (lake) and Stempellina sp.1 (main channel) (Table 3). The main channel was the only habitat where scrapers were recorded (Figure 2B), represented by Phaenopsectra sp.1, thus the diversity of this site was zero.

The detritus included rests of macrophytes, algae, fungi spores, exuviae, and clay. Exuviae of head capsules were recorded only in larvae of the predator $A$. gr. annulata. Besides algae, spores of fungi were recorded sporadically in the predator Procladius sp., and commonly in the collectors-gatherers Fissimentum cf. F. desiccatum and Cricotopus sp.3 and the collector-filterer Tanytarsus sp.

\subsection{Correlations between biotic and abiotic factors}

The sampling sites of the stream produced characteristic negative correlations between some Chironomidae larvae and the water depth: Cricotopus sp.3 ( $\mathrm{r}=-0.925, \mathrm{p}=0.008)$, Caladomyia sp. $(\mathrm{r}=-0.828, \mathrm{p}=0.041)$, and Beardius $\mathrm{cf}$. $B$. parcus $(\mathrm{r}=-0.833, \mathrm{p}=0.039)$. Other significant correlations were found between water transparency and the density of Cricotopus sp.3 ( $\mathrm{r}=-0.953, \mathrm{p}=0.003)$, dissolved oxygen and Tanytarsus sp. $(\mathrm{r}=0.811, \mathrm{p}=0.049), \mathrm{pH}$ and Tanytarsus sp. $(\mathrm{r}=0.927, \mathrm{p}=0.007)$.

No significant correlations were found between biotic and abiotic variables at the sampling sites of the lake, rapids, reservoir, and secondary channel.

Comparing the diversity of the functional feeding groups $(\mathrm{n}=5)$ in the six sampled habitats, similarities were obvious only between the main channel and the stream (correlation: $r=0.900, p=0.037$ ) despite different diversity values.

\subsection{Beta diversity and cluster analysis}

The Beta diversity values between the sampling sites and dietary sources were high, and most of this variation was assigned to spatial turnover (Table 6). Overall, the 
Table 6. Beta diversity analysis with the data of Chironomidae taxa occurrence in the collection sites and the occurrence of food items in the different Chironomidae taxa.

\begin{tabular}{lccc}
\hline \multicolumn{1}{c}{ Analysis data } & Beta Sorensen & Turnover (Beta Simpson) & Residuals (Beta.Sor-Beta.Sim) \\
\hline Sampling sites & 0.94 & 0.83 & 0.11 \\
Dietary sources & 0.94 & 0.89 & 0.05 \\
\hline
\end{tabular}

poorer sampling sites were grouped by the cluster analysis, where there was the occurrence of rarer taxa (Figure 4). Many sampling sites which had high richness did not form clusters and in these places there was the occurrence of more common taxa.

\section{Discussion}

Connectivity to floodplains increase allow an exchange of organic matter and biota between stream and riparian wetlands (Wantzen and Junk, 2000; Junk and Wantzen, 2004) and several components of hydrological connectivity can be distinguished in riverine systems (Wantzen and Junk, 2005; Coops et al., 2008). Within the Sepotuba River, the channels and streams show a lateral connectivity gradient of decreasing exchange between lake water and river water.

Though characteristic for lotic systems (Trivinho-Strixino and Strixino, 2005), Rheotanytarsus was recorded in lakes with muddy ground of the Sepotuba basin. Similarly Butakka (2000) encountered larvae of this genus in floodplain lake close to a channel connecting to the main river (Cuiabá River). Both the systems of communication between the water bodies (floodplain lakes, river channels, permanent connections locally called "corixos") and the degree of connectivity in floodplain systems of the Pantanal can increase the drift of organisms from the channels to the floodplain lakes, generally during inundation periods.

The low diversity of the sites S06 and S35 is caused by the near-exclusive presence of the species Cricotopus sp.3, alone or together with one or another taxa (e.g., Tanytarsus sp. and Djalmabatista sp.3). According to Strixino and Trivinho-Strixino (1998) and Henriques-Oliveira et al. (2000), habitats with rocky substrate and littoral with sand and pebbles in zones of erosion are favourable for the colonisation by larvae of Cricotopus and Corynoneura. These genera contain typical organisms of lotic environments that are rarely encountered in lacustrine systems.

The correlations between the environmental variables and the density of Chironomidae larvae are in accordance with the study of Nessimian et al., (1999), HenriquesOliveira et al. (2003) and Amorim et al. (2004), who found that the sediment type and the water depth were the main factors that influence the species richness and distribution of invertebrates.

The greater presence of Tanytarsus and the lesser presence of Chironomus $\mathrm{cf}$. C. strenzkei in the sampling sites in lakes may indicate an environment with characteristic water quality of high oxygen saturation, low productivity and low eutrophication (Strixino and Trivinho-Strixino, 1998; Marques et al., 1999). Some species of Chironomus prefer or are restricted to water bodies with humic and fulvic substances. Larvae of this genus were encountered in lakes with organic matter and muddy bottom.

From the point of view of the trophic determination one can argue that the associated species showed preference for a certain type of sediment due primarily to the food resources, reflected by the similarity of the diet between the different functional feeding groups.

Nessimian et al. (1999) suggested that correlations between two taxa can prove the common use of biotopes and food resources. The analysis of the digestive content in this study confirms this. These authors found that generalist species and opportunists do not exercise pressure on only one food item (Sanseverino and Nessimian, 2008), but rather have an impact on various food items, confirming that the majority of chironomids are generalists.

Observations of the digestive content from the present study confirm the concept of these authors, as several species swallowed the same algae species, e.g., F. desiccatum and Cricotopus sp.3 (Eunotia, Navicula and Encyonema), Chironomus cf. C. strenzkei and A. gr. annulata (Gomphonema and Pinnularia), and Tanytarsus and Cricotopus sp.3 (Cymbella and Eunotia). These results are valid not only for food resources, but can also reveal spatial relationships, as several species explore the same microhabitats, suggesting that the larva is a non-selective feeder and ingests food items in the proportions they occur in the surrounding water.

Collectors-filterers that constitute a considerable part of the functional groups (Tanytarsus, Rheotanytarsus, Tanytarsus cf. T. obiriciae), consume the dissolved organic matter originating from decomposing plants with high microbial activity, or filter living cells of phytoplankton. According to Trivinho-Strixino and Strixino (1991), organic matter is one of the major food sources in lakes and reservoirs that contribute to the balance of the systems in terms of diversity and richness of faunistic groups.

Marques et al. (1999) confirmed that in genera of Tanypodinae the distribution is limited by the presence of vegetation fragments and algae, by the type of sediment, or by the concentration of nutrients. A. gr. annulata, recorded in channels with sandy bottom with mud feed predominantly on algae. According to Pinder (1986), these larvae have an extremely varied diet of animal and algae items.

The greater diversity in the streams and the main channel lend support to the hypothesis that these habitats play an essential role in the maintenance of diversity of the functional groups of these sites. Heino and Paasivirta (2008) suggested an increase of chironomids diversity from head waters to mid-sized rivers and the patterns shown by the diversity indices that take into account the number of individuals also matched these predicted diversity patterns 

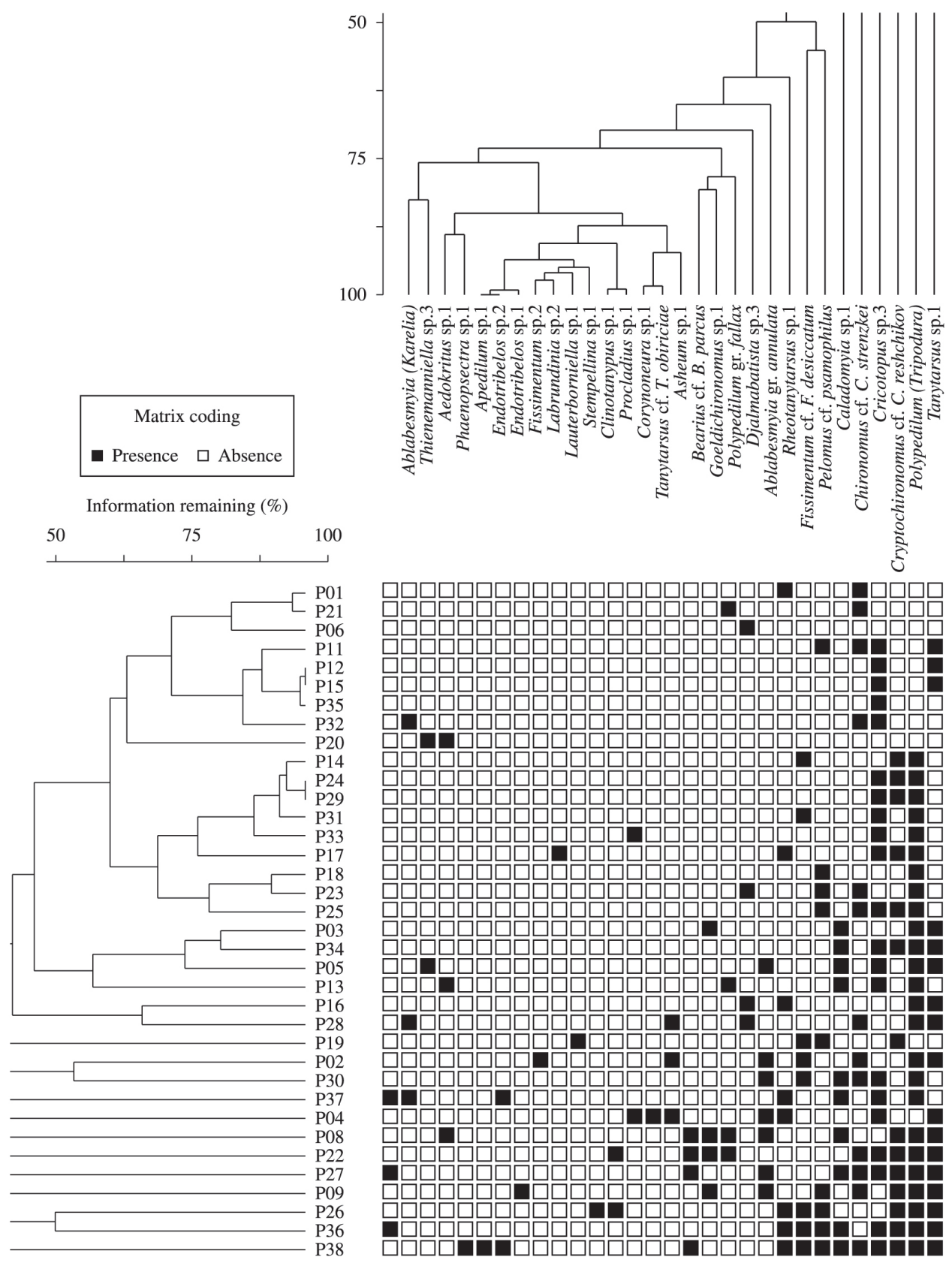

Figure 4. Cluster Dendrogram for Chironomidae occurrence in the collection sites of two-way cluster analysis.

along the river relatively well. However, species density varied primarily with a major water chemistry gradient and not with a stream.

The groups formed from cluster analysis agree with these results, because there was a tendency for grouping of sites in relation to species richness, concurrent with the clustering of taxa in relation to its rarity in the sampling sites. This fact indicates that the morphotypes Chironomidae showed no increase in the richness by the river continuum, concluding that the group responded better to some physical or chemical change of water.
Different habitat characterisations show that some groups indicate preference either for the substrate type or for the habitat type. e.g., larvae of Endotribelos, Goeldichironomus (collectors-gatherers) and Phaenopsectra (scraper) inhabited the sandy bottom of lotic systems. However, Sanseverino and Nessimian (2001) encountered larvae of these genera living preferentially in pockets of litter in areas of deposition and erosion.

In addition, the values of the beta diversity analysis indicate a high variation in the composition of morphotypes between environments. This variation was largely attributed 
to spatial turnover, which allows us to assume that the high dispersal ability of the group has made the species reach the whole region studied. Beta diversity is a wellestablished and widely used concept in ecology and has been measured in different ways and for various purposes (for a review see Tuomisto, 2010), including the turnover, i.e. changes in species composition among local assemblages (Baselga, 2010).

The differences along the river are decisive for the formation of distinct or discontinuous communities, as the changes are more likely in the physical environment, and the limits become obvious though the interrelations between the populations in the community, as for instance, competition for food and habitats, and predation by small fishes.

Thus, variations in the composition and richness among environments can be attributed to local factors governing the occurrence of certain taxa, contributing to compositional dissimilarity between the sampling sites. Moreover our results reveal that these changes may be different for various organisms in accordance with their traits, such as their dispersal abilities.

Acknowledgements - This study received financial support from "Conservation International", and was made possible by the participation of Dra. Alice Michiyo Takeda in the Sepotuba AquaRAP Expedition in 2002, thanks to an invitation of CI. The authors are grateful to the organisers and expedition team of the Sepotuba AquaRAP; and the team of the laboratory of zoobenthos (Nupelia/UEM) for the help in the separation of the larvae, MsC. Josimeire Leandrini for assistance in the identification of algae, chemist Maria do Carmo Roberto for the description of the sampling sites, Dr. Peter Petermann for help with the translation and revision of the manuscript, drawer Jaime Luís Lopes Pereira for the map, and CAPES grant $n^{\circ} 4000$ 4015005 D-5 for the first author.

\section{References}

AB'SABER, AN., 1983. O domínio dos cerrados: Introdução ao conhecimento. Revista do Serviço Público, vol. 111, p. 41-55.

ABURAYA, FH. and CALLIL, CT., 2007. Variação temporal de larvas de Chironomidae (Diptera) no Alto Rio Paraguai (Cáceres, Mato Grosso, Brasil). Revista Brasileira de Zoologia, vol. 24, no. 3, p. 565-572. http://dx.doi.org/10.1590/S0101-81752007000300007.

ALHO, CJR., STRÜSSMANN, C., VOLPE, M., SONODA, F., MARQUES, AAB., SCHNEIDER, M., SANTOS JUNIOR, TS. and MARQUE, SR., 2003. Conservação da biodiversidade da bacia do Alto Paraguai - monitoramento da fauna sob impacto ambiental. Campo Grande: Editora UNIDERP. 449 p.

AMORIM, RM., HENRIQUES-OLIVEIRA, AL. and NESSIMIAN, JL., 2004. Distribuição espacial e temporal das larvas de Chironomidae (Insecta: Diptera) na seção ritral do rio Cascatinha, Nova Friburgo, Rio de Janeiro, Brasil. Lundiana, vol. 5, no. 2, p. 119-127.

BASELGA, A., 2010. Partitioning the turnover and nestedness components of beta diversity. Global Ecology and Biogeography, vol. 19 , no. 1 , p. 134-143. http://dx.doi.org/10.1111/j.14668238.2009.00490.x.
BICUDO, CEM. and BICUDO, RMT., 1970. Algas de águas continentais brasileiras: chave ilustrada para a identificação de gêneros. São Paulo: FUNBEC. 228 p.

BUTAKKA, CMM., 2000. Comunidade de invertebrados bentônicos e características limnológicas da Baía de Sinhá Mariana, Pantanal Mato-grossense, MT. Cuiabá: Universidade Federal do Mato Grosso. 115 p. Master thesis. Instituto de Biociências.

COFFMAN, WP. and FERRINGTON JUNIOR, LC., 2008. Chironomidae. In MERRIT, RW., CUMMINS, KW. and BERG, MB. (Ed.). An introduction to the Aquatic Insects. 4th edition. Dubuque: Kendall Hunt Publishing. p. 551-652.

COOPS, H., BUIJSE, LL., BUIJSE, AD., CONSTANTINESCU, A., COVALIOV, S., HANGANU, J., IBELINGS, BW., MENTING, G., NAVODARU, I., OOSTERBERG, W., STARAS, M. and TÖRÖK, L., 2008. Trophic gradients in a large-river delta: ecological structure determined by connectivity gradients in the DanubE Delta (Romania). River Research and Applications, vol. 24, no. 5, p. 698-709. http://dx.doi.org/10.1002/rra.1136.

DA SILVA, CJ., WANTZEN, KM., CUNHA, CN. and MACHADO, FA., 2001. Biodiversity in the Pantanal Wetland, Brazil. In JUNK, WJ., GOPAL, B. and DAVIS, JA. (Ed.). Biodiversity in Wetlands: assessment, function and conservation. vol. 2. Leiden: Backhuys publishers. p. 187-217.

DE DRAGO, IE., MARCHESE, MR. and WANTZEN, KM., 2004. Benthos of a large neotropical river: spatial patterns and species assemblages in the Lower Paraguay and its floodplains. Archiv fuer Hydrobiologie, vol. 160, no. 3, p. 347-374. http:// dx.doi.org/10.1127/0003-9136/2004/0160-0347.

EPLER, JH., 2001. Identification Manual for the Larval Chironomidae (Diptera) of North and South Carolina. Raleigh: North Carolina Departament of Environmental and Natural Resources Division of Water Quality. 53 p.

HEINO, J. and PAASIVIRTA, L., 2008. Unravelling the determinsnts of stream midge biodiversity in a boreal drainage basin. Freshwater Biology, vol. 53, no. 5, p. 884-896. http://dx.doi.org/10.1111/j.13652427.2007.01946.x.

HENRIQUES-OLIVEIRA, AL., SANSEVERINO, AM. and NESSIMIAN, JL., 2000. Larvas de Chironomidae (Insecta: Diptera) de substrato rochoso em dois rios em diferentes estados de preservação na Mata Atlântica, RJ. Acta Limnologica Brasiliensia, vol. 11 , no. 2 , p. 17-28.

HENRIQUES-OLIVEIRA, AH., NESSIMIAN, JL. and DORVILLÉ, LFM., 2003. Feeding habitats of Chironomid larvae (Insecta: Diptera) from a stream in the Floresta da Tijuca, Rio de Janeiro, Brazil. Revista Brasileira de Biologia, vol. 63, no. 2, p. 16p.

HIGUTI, J. and TAKEDA, AM., 2002. Spatial and temporal variation in desities of Chironomid larvae (Diptera) in two lagoons and two tributaries of the Upper Paraná River floodplain, Brazil. Brazilian Journal of Biology, vol. 62, no. 4b, p. 807-818. http:// dx.doi.org/10.1590/S1519-69842002000500010.

JUNK, WJ. and WANTZEN, KM., 2004. The flood pulse concept: new aspects, approaches, and applications.An Update. In WELCOMME, R. and PETR, T. Proceedings of the 2nd Large River Symposium. Bangkok: FAO Regional Office for Asia and the Pacific/RAP Publication. p. 117-149.

JUNK, WJ., WANTZEN, KM., CUNHA, CN., PETERMANN, P., STRÜSSMANN, C., MARQUES, M. and ADIS, J., 2006. Comparative biodiversity value of large wetlands: the Pantanal of Mato Grosso, Brazil. Aquatic Sciences, vol. 63, p. 278-309. 
KIM, KEC., 1993. Biodiversity, conservation and inventory: why insects matter. Biodiversity and Conservation, vol. 2, no. 3, p. 191-214. http://dx.doi.org/10.1007/BF00056668.

KREMEN, C., COLWELL, RK., ERWIN, TL., MURPHY, DD., NOSS, RF. and SANJAYAN, MA., 1993. Terrestrial arthropod assemblages: their use in conservation planning. Conservation Biology, vol. 7, no. 4, p. 796-808. http://dx.doi.org/10.1046/j.15231739.1993.740796.x.

MAGURRAN, AE.,2004. Measuring Biological Diversity. Australia: Blackwell Publishing Company. 215 p.

MARCHESE, MR., WANTZEN, KM. and DE DRAGO, IE., 2005. Benthic invertebrate assemblages and species diversity patterns of the Upper Paraguay River. River Research and Applications, vol. 21, no. 5, p. 485-499. http://dx.doi.org/10.1002/rra.814.

MARQUES, MMGSM., BARBOSA, FA. and CALLISTO, M., 1999. Distribution and abundance of Chironomidae (Diptera, Insecta) in an impacted watershed in south-east Brasil. Brazilian Journal of Biology, vol. 54, p. 553-561. PMid:23505643.

MCCUNE, B. and MEFFORD, MJ., 1999. PC-ORD. Multivariate Analysis of Ecological Data. Version 5.0 Gleneden Beach: MjM Software.

MERRIT, RW. and WALLACE, BJ., 1981. Insectos filtradores. Investigacion y Ciencia, vol. 57, p. 94-102.

NESSIMIAN, JL., SANSEVERINO, AM. and OLIVEIRA, ALH., 1999. Relações tróficas de larvas de Chironomidae (Diptera) e sua importância na rede alimentar em um brejo no litoral do Estado do Rio de Janeiro. Revista Brasileira de Entomologia, vol. 43, p. 47-53.

OLIVER, I. and BEATTIE, AJ., 1996. Designing a cost-effective invertebrate survey: a test of methods for rapid assessment of biodiversity. Ecological Applications, vol. 6, no. 2, p. 594-607. http://dx.doi.org/10.2307/2269394.

PARRA, OO. and BICUDO, CEM., 1995. Introduction a la Biologia y Sistemática de las Algas de Águas Continentales. Santiago: Andes. 290 p.

PINDER, LCV., 1986. Biology of freshwater Chironomidae. Annual Review of Entomology, vol. 31, no. 1, p. 1-23. http:// dx.doi.org/10.1146/annurev.en.31.010186.000245.

PINDER, LCV., 1995. The habitats of chironomid larvae. In ARMITAGE, PD., CRANSTON, OS. and PINDER, LCV. (Eds.). The Chironomidae: biology and ecology of non-biting midges. London: Chapman \& Hall. p. 107-135.

R Foundation for Statistical Computing, 2011. R: A language and environment for statistical computing. Vienna. Available from: $<$ http://www.R-project.org $>$.

ROQUE, FO. and TRIVINHO-STRIXINO, S., 2003. Guassutanypus oliveirai, a new genus and species of Macropelopiini from Brazil (Insecta, Diptera, Chironomidae). Spixiana, vol. 26, p. 159-164.

SALA, OE., CHAPIN, FSIII 3rd., ARMESTO, JJ., BERLOW, E., BLOOMFIELD, J., DIRZO, R., HUBER-SANWALD, E., HUENNEKE, LF., JACKSON, RB., KINZIG, A., LEEMANS, R., LODGE, DM., MOONEY, HA., OESTERHELD, M., POFF, NL., SYKES, MT., WALKER, BH., WALKER, M. and WALL, DH., 2000. Global biodiversity scenarios for the year 2100. Science, vol. 287 , no. 5459, p. 1770-1774. http://dx.doi.org/10.1126/ science.287.5459.1770. PMid:10710299

SANSEVERINO, AM. and NESSIMIAN, JL., 2001. Hábitats de larvas de Chironomidae (Insecta, Diptera) em riachos de mata Atlântica no Estado do Rio de Janeiro. Acta Limnologica Brasiliensia, vol. 13, p. 29-38.
SANSEVERINO, AM. and NESSIMIAN, JL., 2008. The food of larval chironomids in a stream of the Atlantic Forest (Rio de Janeiro, Brazil). Acta Limnologica Brasiliensia, vol. 20, p. 117-130.

STRIXINO, G. and TRIVINHO-STRIXINO, S., 1998. Povoamentos de Chironomidae (Diptera) em lagos artificiais. In NESSIMIAN, JL. and CARVALHO, AL. (Ed.). Ecologia de Insetos Aquáticos. Rio de Janeiro: Oecologia Brasiliensis 5/PPGE-UFRJ. p. 141-154.

STUR, E., NOLTE, U. and FITTKAU, EJ., 2000. Chironomidae from a surface-drift habitat in an intermittent stream in tropical Brazil. In HOFFRICHTER, O. (Ed.). Late $20^{T H}$ Century research on Chironomidae. Aachen: Shaker Verlag. p. 425-432.

STUR, E., FITTKAU, EJ. and SERRANO, MA., 2006. Parapentaneura bentogomensis gen. n., sp. n., a new Tanypodinae (Diptera, Chironomidae) from Brazil. Zootaxa, vol. 1384, p. 59-68.

TRIVINHO-STRIXINO, S. and STRIXINO, G., 1991. Estrutura da comunidade de insetos aquáticos associados a Pontederia lanceolata Nuttal. Brazilian Journal of Biology, vol. 53, p. 103-111.

TRIVINHO-STRIXINO, S. and STRIXINO, G., 1998. Chironomidae (Diptera) associados a troncos de árvores submersos. Revista Brasileira de Entomologia, vol. 41, p. 173-178.

TRIVINHO-STRIXINO, S., CORREIA, LC. and SONODA, KC., 2000. Phytophilous Chironomidae (Diptera) and other macroinvertebrates in the ox-bow Infernão Lake (Jataí Ecological Station, Luiz Antônio, SP, Brazil). Brazilian Journal of Biology, vol. 60 , no. 3 , p. 527-535.

TRIVINHO-STRIXINO, S. and SANSEVERINO, AM., 2003. Tanytarsus rhabdomantis: New combination for Nimbocera rhabdomantis Trivinho-Strixino \& Strixino, 1991 (Diptera: Chironomidae). Zootaxa, vol. 389, p. 1-10.

TRIVINHO-STRIXINO, S. and STRIXINO, G. 2005., Chironomidae (Diptera) do Rio Ribeira (Divisa dos Estados de São Paulo e Paraná) numa avaliação ambiental faunística. Entomologia y Vectores, vol. 12, no. 2, p. 243-253. http://dx.doi.org/10.1590/ S0328-03812005000200008

TRIVINHO-STRIXINO, S., 2011. Larvas de Chironomidae: guia de identificação. São Carlos: Laboratório de Entomologia Aquática/PPG-ERN/UFSCar.

TUOMISTO, H., 2010. A diversity of beta diversities: straightening up a concept gone awry. Part 1 . Defining beta diversity as a function of alpha and gamma diversity. Ecography, vol. 33, no. 1, p. 2-22. http://dx.doi.org/10.1111/j.1600-0587.2009.05880.x.

WANTZEN, KM. and JUNK, WJ., 2000. The importance of stream-wetland-systems for biodiversity: a tropical perspective. In GOPAL, B., JUNK, WJ. and DAVIES, JA. (Ed.). Biodiversity in Wetlands: assessment, function and conservation Backhuys. Leiden: The Netherlands. p. 11-34.

WANTZEN, KM. and JUNK, WJ., 2005. Aquatic-terrestrial linkages from streams to rivers: biotic hot spots and hot moments. Archiv für Hydrobiologie Supplements, vol. 158, p. 595-611.

WANTZEN, KM. and WAGNER, R., 2006. Detritus processing by invertebrate shredders: a neotropical-temperate comparison. Journal of the North American Benthological Society, vol. 25, no. 1, p. 216-230. http://dx.doi.org/10.1899/0887-3593(2006)25[216:DPBISA]2.0.CO;2.

WENTWORTH, CK., 1922. A scale of grade and class trems for clastic sediments. The Journal of Geology, vol. 30, no. 5, p. 377-392. http://dx.doi.org/10.1086/622910.

YEN, AL. and BUTCHER, RJ., 1997. An overview of the conservation of non-marine invertebrates in Australia. Canberra: Environment Australia. 